\title{
Optimization of Structural Design by Fuzzy Geometric Programming Technique
}

\author{
Mridula Sarkar ${ }^{1}$ Samir Dey ${ }^{2 *}$ Tapan Kumar Roy ${ }^{3}$ \\ ${ }^{1,3}$ Department of Mathematics, Indian Institute of Engineering Science and Technology, Shibpur. P.O.-Botanic \\ Garden, Howrah-711103, West Bengal, India. \\ ${ }^{2}$ Department of Mathematics, Asansol Engineering College Vivekananda Sarani, Asansol-713305, West Bengal, \\ India.
}

\begin{abstract}
In this paper we develop solution procedure of fuzzy geometric programming based on Warner's approach for optimizing the design of plane truss structure with single objective subject to a specified constraint. In this optimum design formulation, the objective function is the weight of the truss; the design variables are the cross-sections of the truss members; the constraint is the stresses in member. A classical truss optimization example is presented here in to demonstrate the efficiency of this technique. The test problem includes a two-bar planar truss subjected to a single load condition. This single-objective structural optimization model is solved fuzzy geometric programming technique. Numerical example is given to illustrate our approach.
\end{abstract}

Keyword:Two-bar Truss Design, GeometricProgramming,Fuzzy Geometric Programming, Warner'sApproach, Max-additive Operator.

\section{Introduction}

The research area of optimal structural design has been receiving increasing attention fromboth academic and industry for past three decades in order to improve structural performance and to reduce design costs. However, in the real world, uncertainty or vagueness is prevalent in the Engineering Computations .Commonly such uncertainties are included in the design process by introducing simplified hypothesis and safety or design factors. This tendency has been changing due to the increase in the use of different fuzzy mathematical algorithm for dealing with this class of problems. Zadeh[1] first introduced the concept of fuzzy set theory. Then Zimmermann [2] applied the fuzzy set theory concept with some suitable membership functions to solve linear programming problem with several objective functions. Several researchers like Wang et al. [3] first applied $\alpha$-cut method to structural designs where the non-linear problems were solved with various design levels $\alpha$, and then a sequence of solutions were obtained by setting different level-cut value of $\alpha$. Rao[4] applied the same $\alpha$-cut method to design a four-bar mechanism for function generating problem. Structural optimization with fuzzy parameters was developed by Yeh et al. [5] used two-phase method for fuzzy optimization of structures. Shih et al. [6] used level-cut approach of the first and second kind for structural design optimization problems with fuzzy resources. Dey et al. [7] used generalized fuzzy number in context of a structural design. Also Dey et al.[8] used basic t-norm based fuzzy optimization technique for optimization of structure.

Geometric Programming (GP) method is an effective method used to solve a non-linear programming problem like structural problem. It has certain advantages over the other optimization methods. Here, the advantage is that it is usually much simpler to work with the dual than the primal one. Solving a non-linear programming problem by GP method with degree of difficulty (DD) plays essential role. (It is defined as DD = total number of terms in objective function and constraints - total number of decision variables -1).

Since late 1960's, GP has been known and used in various fields (like OR, Engineering sciences etc.). Duffin et al. [9]discussed the basic theories on GP with engineering application in their books. Another famous book on GP and its application appeared in 1976 (Beightler et al., [10].The most remarkable property of GP is that a problem with highly nonlinear constraints can be transformed equivalently into a problem with only linear constraints. In real life, there are many diverse situations due to uncertainty in judgments, lack of evidence etc. Sometimes it is not possible to get relevant precise data for the cost parameter. The idea of impreciseness (fuzziness) in GP i.e. fuzzy geometric programming was proposed by Cao [11]. Yang et al. [12] discussed about the basic and its applications of fuzzy geometric programming. A solution method of posynomial geometric programming with interval exponents and coefficients was developed by Liu [13]. Ojha et al. [14] used binary number for splitting the cost coefficients, constraints coefficient and exponents and then solved it by GP technique. Deyet.al[15]Optimized shape design of structural model with imprecise coefficient by parametric geometric programming.Nasseri et al. [16] solved two bar truss nonlinear problem by using geometric 
programming technique into the form of two-level mathematical programming.In this paper we find optimum weight with optimum angle of two bar truss by additive aggregation following warner's approach.

\section{Single-objective Structural Model}

In sizing optimization problems, the aim is to minimize a single objective function, usually theweight of the structure, under certain behavioural constraints on stress and displacements. The design variables are most frequently chosen to be dimension of the cross sectional areas of the members of the structure.Due to fabrication limitations the design variables are not continuous but discrete since cross sections belong to a certain set.A discrete structural optimization problem can be formulated in the following form

Minimize $f(A)$

$$
\begin{gathered}
\text { subject to } g_{i}(A) \quad i=1,2, \ldots \ldots ., m \text { (1) } \\
A_{j} \in R^{d}, j=1,2, \ldots \ldots ., n
\end{gathered}
$$

where $f(A)$ represents objective function, $g(A)$ is the behavioural constraint, $m$ and $n$ are the number of constraints and design variables, respectively. A given set of discrete values is expressed by $R^{d}$ and design variables $A_{j}$ can take values only from this set.In this paper,objective function is taken as

$$
f(A)=\sum_{i=1}^{m} \rho_{i} A_{i} l_{i}
$$

and constraints are chosen to be stress of structures as follows

$$
g_{i}(A)=\frac{\sigma_{i}}{\sigma_{i}^{0}}-1 \leq 0 \quad i=1,2, \ldots \ldots \ldots . ., m
$$

Where $\rho_{i}$ and $l_{i}$ are weight of unit volume and length of $i^{t h}$ element respectively. $\mathrm{m}$ is the number of the structural elements $\sigma_{i}$ and $\sigma_{i}^{0}$ are the $i^{\text {th }}$ stress and allowable stressrespectively.

\subsection{Fuzzy Set}

\section{Mathematical preliminaries}

Afuzzy set $\tilde{A}$ in a universe of discourse $\mathrm{X}$ is defined as the following set of pairs $\tilde{A}=\left\{\left(x, \mu_{\tilde{A}}(x)\right): x \in X\right\}$. Here $\mu_{\tilde{A}}: X \rightarrow[0,1]$ is a mapping called the membership value of $x \in X$ in a fuzzy set $\tilde{A}$.

\section{2. $\alpha$-Cut of Fuzzy Set}

The $\alpha$-level set (or interval of confidence at level $\alpha$ or $\alpha$-cut) of the fuzzy set $\tilde{A}$ of $\mathrm{X}$ is a crisp set $A_{\alpha}$ that contains all the elements of $\mathrm{X}$ that have membership values in $\mathrm{A}$ greater than or equal to $\alpha$ i.e. $A_{\alpha}=\left\{x: \mu_{\tilde{A}}(x) \geq \alpha, x \in X, \alpha \in[0,1]\right\}$.

\subsection{Fuzzy Decision Making}

In this real world most of the decision making problem takes place in a fuzzy environment. The objective goal, constraints and consequences of possible actions are not known precisely. Under this observation, Bellman et al. [12] introduced three basic concepts. They are fuzzy objective goal, fuzzy constraint and fuzzy decision based on fuzzy goal and constraint. We introduce the conceptual framework for decision making in a fuzzy environment .Let $X$ be a given set of possible alternatives which contains the solution of a decision making problem in fuzzy environment. The problem based on fuzzy decision making may be considered as follows

Optimize fuzzy goal $\tilde{G}$

Subjet to constraint $\tilde{C}$

A Fuzzy goal $\tilde{G}=\left\{\left(x, \mu_{\tilde{G}}(x)\right) \mid x \in X\right\}$ and a fuzzy constraint $\tilde{C}=\left\{\left(x, \mu_{\tilde{C}}(x)\right) \mid x \in X\right\}$ is a fuzzy set characterised by its membership function $\mu_{\tilde{G}}(x): X \rightarrow[0,1]$ and $\mu_{\tilde{C}}(x): X \rightarrow[0,1]$ respectively. Both 
the fuzzy goal and fuzzy constraint are desired to be satisfied simultaneously, So Bellman et al. [3] defined fuzzy decision through fuzzy goal and fuzzy constraint.

\subsection{Additive Fuzzy Decision}

Fuzzy decision based on additive operator is a fuzzy set $\tilde{D}_{a}=\left\{\left(x, \mu_{\tilde{D}}(x)\right) \mid x \in X\right\}$ such that $\tilde{D}_{a}=\mu_{\tilde{G}}(x)+\mu_{\tilde{C}}(x)$ for all $x \in X$.

\subsection{Single-objective Geometric Programming Problem}

\section{Mathematical Analysis}

A single-objective geometric programming problem can be defined as

Find $x=\left(x_{1}, x_{2}, x_{3}, \ldots \ldots x_{n}\right)^{T}$ so as to

Minimize $f_{0}(x)=\sum_{t=1}^{T_{0}} \varepsilon_{0 t} c_{0 t} \prod_{j=1}^{n} x_{j}^{a_{0 t j}}$

such that $f_{i}(x)=\sum_{t=1}^{T_{i}} \varepsilon_{i t} c_{i t} \prod_{j=1}^{n} x_{j}^{a_{i j}} \leq \varepsilon_{i}$

$x_{j}>0 \quad$ for $i=1,2, \ldots \ldots \ldots .$, mand $j=1,2, \ldots \ldots \ldots, n$

Here $\varepsilon_{0 t}, \varepsilon_{i t}, \varepsilon_{i}$ are signum functions used to indicate sign of terms in the equation. $c_{0 t}>0, c_{i t}>0, a_{i t j}, a_{0 t j}$ are all real numbers for all $i, t, j$

Here Degree of Difficulty of (4) i.e $D D_{\text {SOGPP }}=T_{0}+\sum_{i=1}^{m} T_{i}-(n+1)$

The dual formulation of the above primal geometric programming problem is

$\operatorname{maximize} d_{\text {SOGPP }}=\varepsilon\left[\prod_{i=0}^{m} \prod_{t=1}^{T_{i}}\left(\frac{c_{i t} w_{i 0}}{w_{i t}}\right)^{\varepsilon_{i t} w_{i t}}\right]^{\varepsilon}$ for $i=0,1,2, \ldots \ldots \ldots, m$

such that $w_{00}=1$

$\sum_{t=1}^{T_{0}} \varepsilon_{0 t} w_{0 t}=\varepsilon$

$\sum_{i=0}^{m} \sum_{t=1}^{T_{i}} \varepsilon_{i t} a_{i t j} w_{i t}=0 \quad$ for $j=1,2, \ldots \ldots \ldots ., n$

$w_{i t} \geq 0$ for $i=1,2, \ldots \ldots \ldots . . m ; t=1,2, \ldots \ldots \ldots . T_{i}$

$w_{i 0}=\varepsilon_{i} \sum_{t=1}^{T_{i}} \varepsilon_{i t} w_{i t} \geq 0 \quad$ for $i=1,2, \ldots \ldots \ldots \ldots . . ., m$

From primal-dual relations

$c_{0 t} \prod_{j=1}^{m} x_{j}^{a_{0 t j}}=w_{0 t} \varepsilon d_{\text {SOGPP }}^{*}\left(w^{*}\right) \quad$ for $t=1,2, \ldots \ldots ., T_{0}$

$c_{i t} \prod_{j=1}^{n} x_{j}^{a_{i j}}=\frac{w_{i t}}{w_{i 0}} \quad$ for $t=1,2, \ldots \ldots \ldots ., T_{i} \quad i=1,2, \ldots \ldots \ldots, m$

we will get optimal primal decision variables and corresponding objective values.

\subsection{Single-objective Fuzzy Geometric Programming Problem}

In this section we discuss the optimization problem with non-linear fuzzy objective and fuzzy nonlinear constraints. Consider the following geometric programming problem

Find $x=\left(x_{1}, x_{2}, x_{3}, \ldots \ldots x_{n}\right)^{T}$ so as to 
Minimize $f_{0}(x)=\sum_{t=1}^{T_{0}} \varepsilon_{0 t} c_{0 t} \prod_{j=1}^{n} x_{j}^{a_{0 t j}}$

such that $f_{i}(x)=\sum_{t=1}^{T_{i}} \varepsilon_{i t} c_{i t} \prod_{j=1}^{n} x_{j}^{a_{i j}} \leq b_{i}$ for $i=1,2, \ldots \ldots \ldots . ., m$

$$
x_{j}>0 \quad \text { for } j=1,2, \ldots \ldots \ldots, n
$$

The fuzzy version of the problem (4)is

Minimize $f_{0}(x)=\sum_{t=1}^{T_{0}} \varepsilon_{0 t} c_{0 t} \prod_{j=1}^{n} x_{j}^{a_{0 t j}}$

such that $f_{i}(x)=\sum_{t=1}^{T_{i}} \varepsilon_{i t} c_{i t} \prod_{j=1}^{n} x_{j}^{a_{i j}} \lesssim b_{i}$ for $i=1,2, \ldots \ldots \ldots . ., m$

$x_{j}>0$

for $j=1,2$, .

In problem (5) tilde sign denotes the fuzzy satisfaction of the constraints.Clearly the constraints are flexible in nature.Thefuzzy minimize will be achieved for corresponding lowest possible aspiration level for the general $f_{0}(x)$. This problem can be solved by fuzzy decision making as follows

Step I : Fuzzyfy the objective function by calculating the lower and upper bound of the optimal values. Solve the single objective geometric programming problem without tolerance in constraints (i.e $f_{i}(x) \leq b_{i}$ for $\left.i=1,2, \ldots \ldots \ldots . . ., m\right) \quad$ and tolerance of acceptance in constraints (i.e $f_{i}(x) \leq b_{i}+p_{i}$ for $\left.i=1,2, \ldots \ldots \ldots . ., m\right)$ by fuzzy geometric programming.

Here they are

Sub-problem-1

Find $x=\left(x_{1}, x_{2}, x_{3}, \ldots \ldots x_{n}\right)^{T}$ so as to

Minimize $f_{0}(x)=\sum_{t=1}^{T_{0}} \varepsilon_{0 t} c_{0 t} \prod_{j=1}^{n} x_{j}^{a_{0 t j}}(6)$

such that $f_{i}(x)=\sum_{i=1}^{T_{i}} \varepsilon_{i t} c_{i t} \prod_{j=1}^{n} x_{j}^{a_{i j}} \leq b_{i}$ for $i=1,2, \ldots \ldots \ldots . ., m$

$x_{j}>0 \quad$ for $j=1,2, \ldots \ldots \ldots, n$

Here $\varepsilon_{0 t}, \varepsilon_{i t}, \varepsilon_{i}$ are signum functions used to indicate sign of terms in the equation. $c_{0 t}>0, c_{i t}>0, a_{i t j}, a_{0 t j}$ are all real numbers for all $i, t, j$.

And

Sub-problem-2

Find $x=\left(x_{1}, x_{2}, x_{3}, \ldots . . x_{n}\right)^{T}$ so as to

Minimize $f_{0}(x)=\sum_{t=1}^{T_{0}} \varepsilon_{0 t} c_{0 t} \prod_{j=1}^{n} x_{j}^{a_{0 t j}}$

such that $f_{i}(x)=\sum_{t=1}^{T_{i}} \varepsilon_{i t} c_{i t} \prod_{j=1}^{n} x_{j}^{a_{i j}} \leq b_{i}+p_{i}$ for $i=1,2, \ldots \ldots \ldots . ., m$

$x_{j}>0 \quad$ for $j=1,2, \ldots \ldots \ldots ., n$

Here $\varepsilon_{0 t}, \varepsilon_{i t}, \varepsilon_{i}$ are signum functions used to indicate sign of terms in the equation. $c_{0 t}>0, c_{i t}>0, a_{i t j}, a_{0 t j}$ are all real numbers for all $i, t, j$. 
Solving (6) and (7) we may get optimal solutions $x^{*}=x^{1}, f_{0}\left(x^{*}\right)=f\left(x^{1}\right)$ and $x^{*}=x^{2}, f_{0}\left(x^{*}\right)=f\left(x^{2}\right)$ respectively. Now wefind the lower bound(minimum) $L$ and upper bound (maximum) $U$ by using following rule $U=\max \left\{f_{0}\left(x^{1}\right), f_{0}\left(x^{2}\right)\right\}$ and $L=\min \left\{f_{0}\left(x^{1}\right), f_{0}\left(x^{2}\right)\right\}$.

Suppose $\mathrm{M}$ be the fuzzy set representing the objective function such that $M=\left\{x \in R^{n}:\left(x, \mu_{M}(x)\right)\right\}$ where $\mu_{M}(x)$ is defined as

$$
\mu_{M}(x)= \begin{cases}1 & \text { if } f_{0}(x) \leq L \\ \frac{U-f_{0}(x)}{U-L} & \text { if } L \leq f_{0}(x) \leq U \\ 0 & \text { if } f_{0}(x) \geq U\end{cases}
$$

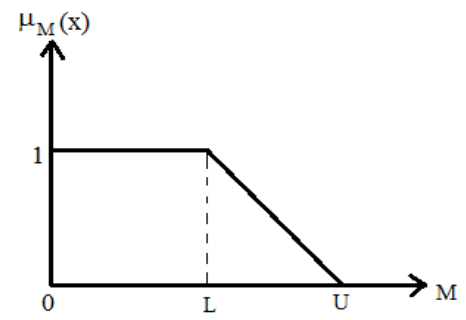

Fig.1. Rough sketch of membership function for objective

Step II :Fuzzyfy the constraint $f_{i}(x)$ for $i=1,2, \ldots, m$. Let $C_{i}$ be the fuzzy set for jth constraints such that $C_{i}=\left\{x \in R^{n}:\left(x, \mu_{C_{i}}(x)\right)\right\}$, where $\mu_{C_{i}}(x)$ is defined as

$$
\mu_{C_{i}}(x)=\left\{\begin{array}{cl}
1 & \text { if } f_{i}(x) \leq b_{i} \\
\frac{b_{i}+p_{i}-f_{i}(x)}{p_{i}} & \text { if } b_{i} \leq f_{i}(x) \leq b_{i}+p_{i} \\
0 & \text { if } f_{i}(x) \geq b_{i}+p_{i}
\end{array}\right.
$$

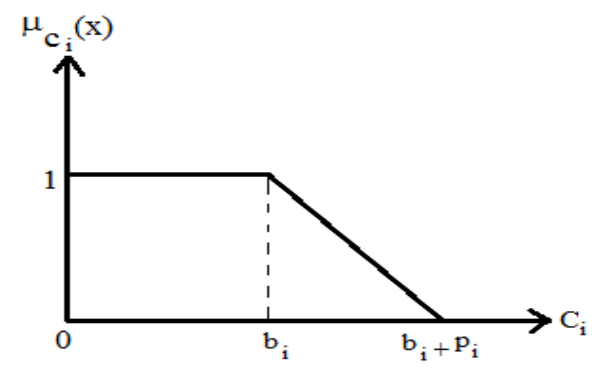

Fig.2. Rough sketch of membership function for constraints

According to fuzzy decision making process [3] on fuzzy objective and constraint goals using membership function (10) and (11) the problem of finding maximizing decision to chose optimal $x^{*}$ using max-additive operator is presented as follows

Find $x^{*}=\left(x_{1}^{*}, x_{2}^{*}, x_{3}^{*}, \ldots \ldots x_{n}^{*}\right)^{T}$ where

$\mu_{D}\left(x^{*}\right)=\max _{x_{j}>0, j=1,2, \ldots, n}\left(\sum_{i=0}^{m} \mu_{i}\left(f_{i}(x)\right)\right)$

Consider the membership function (10) the problem (8) reduces to 
$\operatorname{Max} V_{a}(x)=\sum_{i=0}^{m} \frac{b_{i}+p_{i}}{p_{i}}-V_{a}^{\prime}\left(x^{*}\right)$

Where $x^{*}=\left(x_{1}^{*}, x_{2}^{*}, x_{3}^{*}, \ldots \ldots x_{n}^{*}\right)^{T}$ is optimal decision variable vector of the unconstrained GP problem

$\operatorname{Min} V_{a}^{\prime}(x)=\sum_{i=0}^{m} \frac{1}{p_{i}} \sum_{i=1}^{T_{i}} c_{i t} \prod_{j=1}^{n} x^{a_{i j}}$

subject to $x_{j}>0$ for $j=1,2, \ldots \ldots \ldots \ldots, n$

Now problem (9) is an unconstrained posynomialGP problem with $D D=\sum_{i=0}^{m} T_{i}-(n+1)$ It can be solved by GP technique.

\section{Application on Structural Model}

A well-known two bar planer truss is considered here.The design objective is to minimize weight of the structure $W T\left(A_{1}, A_{2}\right)$ and minimize the deflection $\delta\left(A_{1}, A_{2}\right)$ along $x$ and $y$ axis at a loading point of a statistically loaded three bar planer truss subject to stress constraints on each of the truss members

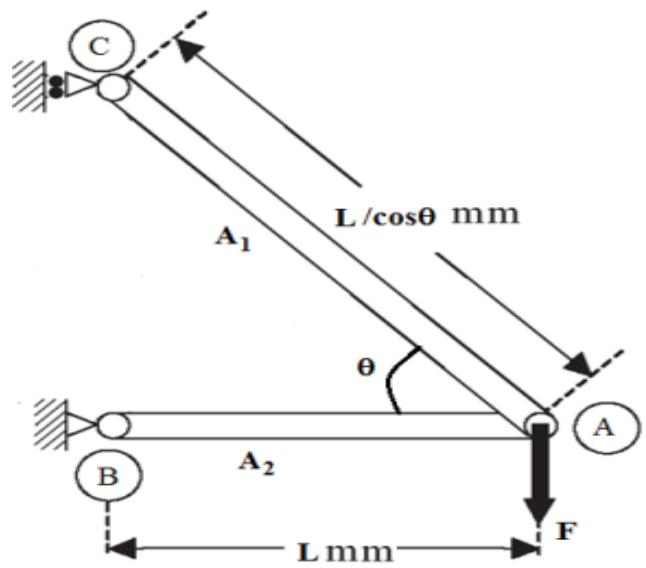

Fig.3. Rough sketch of two-bar truss design

The optimization model of two bar truss is as follows

Minimize $W T\left(A_{1}, A_{2}\right)=\rho L\left(\frac{A_{1}}{\cos \theta}+A_{2}\right)$

$$
\begin{gathered}
\text { subject to } \sigma\left(A_{1}, A_{2}\right) \equiv \frac{F}{A_{1} \cos \theta \sin ^{2} \theta}+\frac{F \cos ^{2} \theta}{A_{2} \sin ^{2} \theta} \leq\left[\sigma_{0}^{T}\right] \\
A_{1}, A_{2}>0 ; \theta_{1}, \theta_{2} \in\left(0,90^{\circ}\right)
\end{gathered}
$$

Where weight $=W T$, Nodal load $=F$, Volume density $=\rho$, Length $A C=L$, Length $A B=L / \cos \theta$, Cross sectional area of bar $A C=A_{1}$ andCross sectional area of bar $A B=A_{2}$.

The input data for structural optimization problem (10) is given

Table 1. Input data of two bar truss design problem

\begin{tabular}{|l|l|l|l|l|l|}
\hline $\begin{array}{l}\text { Young Modulus } \\
\mathrm{E}\left(\mathrm{N} / \mathrm{mm}^{2}\right)\end{array}$ & $\begin{array}{l}\text { Density } \\
\rho\left(\mathrm{N} / \mathrm{mm}^{3}\right)\end{array}$ & $\begin{array}{l}\text { Length of the bar } \\
\mathrm{L}(\mathrm{mm})\end{array}$ & $\begin{array}{l}\text { Applied force } \\
\mathrm{F}(\mathrm{N})\end{array}$ & $\begin{array}{l}\text { Stress } \\
\sigma_{0}\left(\mathrm{~N} / \mathrm{mm}^{2}\right)\end{array}$ & $\begin{array}{l}\text { Maximum } \\
\text { allowable } \\
\text { Stress } \\
\sigma_{0}^{\prime}\left(\mathrm{N} / \mathrm{mm}^{2}\right)\end{array}$ \\
\hline $2 \times 10^{5}$ & $7850 \times 10^{-8}$ & 2500 & 30000 & 200 & 50 \\
\hline
\end{tabular}


Now fuzzy version of the problem is

Minimize $W T\left(A_{1}, A_{2}\right)=.19625\left(\frac{A_{1}}{\cos \theta}+A_{2}\right)$

subject to $\sigma\left(A_{1}, A_{2}\right) \equiv \frac{30000}{A_{1} \cos \theta \sin ^{2} \theta}+\frac{30000 \cos ^{2} \theta}{A_{2} \sin ^{2} \theta} \leq 200$ with tolerance 50

$A_{1}, A_{2}>0 ; \theta \in\left(0,90^{\circ}\right)$

Therefore $b_{1}=200$ and $p_{1}=50$.Now we can find lower bound $L$ and upper bound $U$ by solvingtwo crisp geometric programming problem as follows

Sub-problem-1

Minimize $W T\left(A_{1}, A_{2}\right)=.19625\left(\frac{A_{1}}{\cos \theta}+A_{2}\right)$

subject to $\sigma\left(A_{1}, A_{2}\right) \equiv \frac{30000}{A_{1} \cos \theta \sin ^{2} \theta}+\frac{30000 \cos ^{2} \theta}{A_{2} \sin ^{2} \theta} \leq 200$;

$A_{1}, A_{2}>0 ; \theta \in\left(0,90^{\circ}\right)$

Appling geometric programming technique the optimal solutionis $W T_{1}^{*}=2.37 \times 10^{2} \mathrm{~N}$.

Sub-problem-2

Minimize $W T\left(A_{1}, A_{2}\right)=.19625\left(\frac{A_{1}}{\cos \theta}+A_{2}\right)$

subject to $\sigma\left(A_{1}, A_{2}\right) \equiv \frac{30000}{A_{1} \cos \theta \sin ^{2} \theta}+\frac{30000 \cos ^{2} \theta}{A_{2} \sin ^{2} \theta} \leq 250$;

$A_{1}, A_{2}>0 ; \theta \in\left(0,90^{0}\right)$

Appling geometric programming technique the optimal solution is $W T_{2}^{*}=1.89 \times 10^{2} \mathrm{~N}$.

Therefore $U=\max \left\{W T_{1}^{*}, W T_{2}^{*}\right\}$ and $L=\min \left\{W T_{1}^{*}, W T_{2}^{*}\right\}$.Let $M$ be the set of objective function such that $M=\left\{A_{1}, A_{2} \in R^{n}:\left(x, \mu_{M}\left(A_{1}, A_{2}\right)\right)\right\}$ where

$$
\mu_{M}\left(A_{1}, A_{2}\right)=\left\{\begin{array}{cl}
1 & \text { if } W T\left(A_{1}, A_{2}\right) \leq 1.89 \times 10^{2} \\
\frac{2.37 \times 10^{2}-W T\left(A_{1}, A_{2}\right)}{0.48 \times 10^{2}} & \text { if } 1.89 \times 10^{2} \leq W T\left(A_{1}, A_{2}\right) \leq 2.37 \times 10^{2} \\
0 & \text { if } W T\left(A_{1}, A_{2}\right) \geq 2.37 \times 10^{2}
\end{array}\right.
$$

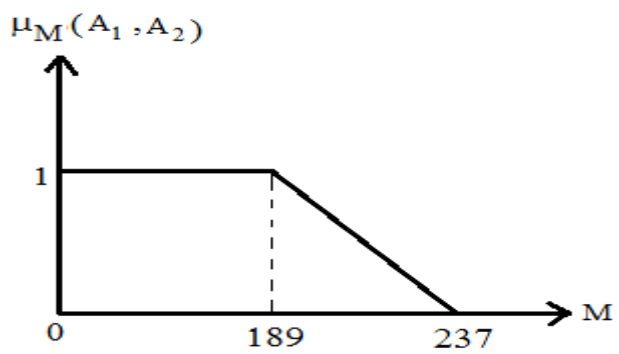

Fig.4. Rough sketch of membership function for weight function

In addition, let $C_{1}$ be the set of constraint such that $C_{1}=\left\{A_{1}, A_{2} \in R^{n}:\left(x, \mu_{C_{1}}\left(A_{1}, A_{2}\right)\right)\right\}$ 
Where $\mu_{C_{1}}\left(A_{1}, A_{2}\right)=\left\{\begin{array}{cl}1 & \text { if } \sigma\left(A_{1}, A_{2}\right) \leq 200 \\ \frac{250-\sigma\left(A_{1}, A_{2}\right)}{50} & \text { if } 200 \leq \sigma\left(A_{1}, A_{2}\right) \leq 250 \\ 0 & \text { if } \sigma\left(A_{1}, A_{2}\right) \geq 250\end{array}\right.$

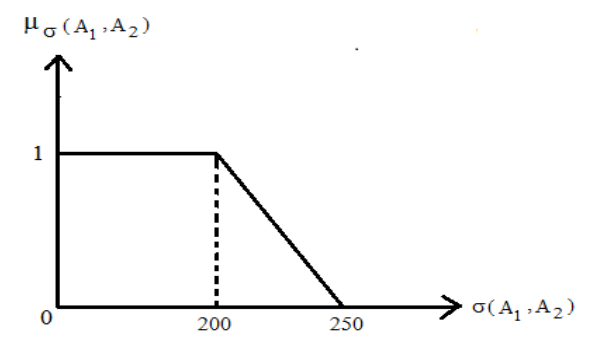

Fig.5. Rough sketch of membership function for constraint function

Now using additive operator fuzzy geometric programming problem can be formulated as

$$
\begin{gathered}
\text { Maximize } W T\left(A_{1}, A_{2}\right)=\frac{2.37 \times 10^{2}-W T\left(A_{1}, A_{2}\right)}{0.48 \times 10^{2}}-\frac{250 \times 10^{2}-\sigma\left(A_{1}, A_{2}\right)}{50} \\
A_{1}, A_{2}>0
\end{gathered}
$$

Neglecting constant term the problem can be formulated as

Maximize $W T\left(A_{1}, A_{2}\right)=P A_{1}+Q A_{1}^{-1}+R A_{2}+S A_{2}^{-1}$

$$
A_{1}, A_{2}>0
$$

Where $P=\frac{4.03 \times 10^{-3}}{\cos \theta} ; Q=\frac{0.6}{\sin ^{2} \theta \cos \theta} ; R=4.09 \times 10^{-3} ; S=\frac{0.6 \cos ^{2} \theta}{\sin ^{2} \theta}$

It is a posynomial primal Geometric Problem (PGP) with $D D=4-(2+1)=1$ and its dual Geometric programming problem (DGP) is

$$
\begin{aligned}
& \text { Maximize } d_{W T_{M A}}\left(w_{01}, w_{02}, w_{03}, w_{04}\right)=\left(\frac{P}{w_{01}}\right)^{w_{01}}\left(\frac{Q}{w_{02}}\right)^{w_{02}}\left(\frac{R}{w_{03}}\right)^{w_{03}}\left(\frac{S}{w_{04}}\right)^{w_{04}} \\
& w_{01}+w_{02}+w_{03}+w_{04}=1, \\
& w_{01}-w_{02}=0 \\
& w_{01}, w_{02}, w_{03}, w_{04}>0
\end{aligned}
$$

Here the constraints of (16) are formed as system of three linear equation with four unknowns. Therefore the system has an infinite number of solutions. However the problem is to select the optimal solution of the dual variables $w_{01}, w_{02}, w_{03}, w_{04}$.

We have from constraint of (16)

$$
\begin{aligned}
& w_{01}=0.5-w_{04}, \\
& w_{02}=0.5-w_{04}, \\
& w_{01}=0.5-w_{04},
\end{aligned}
$$

Substituting $w_{01}, w_{02}, w_{03}$ in (17) we get

$$
\text { Maximize } d_{W T}\left(w_{04}\right)=\left(\frac{P}{0.5-w_{04}}\right)^{\left(0.5-w_{04}\right)}\left(\frac{Q}{0.5-w_{04}}\right)^{\left(0.5-w_{04}\right)}\left(\frac{R}{w_{04}}\right)^{w_{04}}\left(\frac{S}{w_{04}}\right)^{w_{04}}
$$

To find the optimal $w_{04}$ which maximizes the dual $d_{W T_{M A}}\left(w_{04}\right)$ we take logarithm of both side of (17) and get 
$\log d_{W T}\left(w_{04}\right)=\left(0.5-w_{04}\right) \log P-\left(0.5-w_{04}\right) \log \left(0.5-w_{04}\right)+\left(0.5-w_{04}\right) \log Q-\left(0.5-w_{04}\right) \log \left(0.5-w_{04}\right)+$ $w_{04} \log R-w_{04} \log w_{04}+w_{04} \log S-w_{04} \log w_{04}$

Differentiating partially both sides of $d_{W T}\left(w_{04}\right)$ with respect to $w_{04}$ and equating to zero we get

$$
\frac{\left(0.5-w_{04}\right)^{2} S R}{w_{04}^{2} P Q}=1(18)
$$

Here

$$
\frac{\partial^{2} \log d_{W T}\left(w_{04}\right)}{\partial^{2} w_{04}}=\frac{1}{\left(0.5-w_{04}\right) w_{04}}<0\left[\text { as } w_{01}=0.5-w_{04} ; w_{01}, w_{04}>0\right. \text { ] }
$$

So (17) and) (18) gives us optimal dual variables $w_{01}^{*}, w_{02}^{*}, w_{03}^{*}, w_{04}^{*}$

From the primal dual relations

$$
\begin{aligned}
& P A_{1}=w_{01}^{*} d^{*}\left(w_{01}^{*}, w_{02}^{*}, w_{03}^{*}, w_{04}^{*}\right), \\
& Q A_{1}^{-1}=w_{02}^{*} d^{*}\left(w_{01}^{*}, w_{02}^{*}, w_{03}^{*}, w_{04}^{*}\right), \\
& R A_{2}=w_{03}^{*} d^{*}\left(w_{01}^{*}, w_{02}^{*}, w_{03}^{*}, w_{04}^{*}\right), \\
& S A_{2}^{-1}=w_{04}^{*} d^{*}\left(w_{01}^{*}, w_{02}^{*}, w_{03}^{*}, w_{04}^{*}\right)
\end{aligned}
$$

\begin{tabular}{|c|c|c|c|c|}
\hline $\begin{array}{l}\text { Angle between } \\
\text { two-bar }(\theta)\end{array}$ & $\begin{array}{l}\text { Optimum dual } \\
\text { Variables } \\
w_{01}^{*}, w_{02}^{*}, w_{03}^{*}, w_{04}^{*}\end{array}$ & $\begin{array}{l}\text { Optimum cross- } \\
\text { sectional area of } \\
\text { first bar } \\
A_{1}^{*}(\mathrm{~mm})\end{array}$ & $\begin{array}{l}\text { Optimum cross- } \\
\text { sectional area of } \\
\text { first bar } \\
A_{2}^{*}(\mathrm{~mm})\end{array}$ & $\begin{array}{l}\text { Optimum weight of } \\
\text { the two-bar truss } \\
W T^{*}\left(\mathrm{Kg} / \mathrm{mm}^{2}\right)\end{array}$ \\
\hline $35^{\circ}$ & $\begin{array}{l}w_{01}^{*}=.2991332, w_{02}^{*}=.2991332 \\
w_{03}^{*}=.2008668, w_{04}^{*}=.2008668\end{array}$ & 194.2545 & 159.0124 & $.7774509 \times 10^{2}$ \\
\hline $40^{\circ}$ & $\begin{array}{l}w_{01}^{*}=.3150893, w_{02}^{*}=.3150893 \\
w_{03}^{*}=.1849107, w_{04}^{*}=.1849107\end{array}$ & 48.66622 & 37.27872 & $.1978356 \times 10^{2}$ \\
\hline $45^{\circ}$ & $\begin{array}{l}w_{01}^{*}=.3332370, w_{02}^{*}=.3332370 \\
w_{03}^{*}=.1667630, w_{04}^{*}=.1667630\end{array}$ & 41.03149 & 28.98855 & $.1707686 \times 10^{2}$ \\
\hline $50^{\circ}$ & $\begin{array}{l}w_{01}^{*}=.3538262, w_{02}^{*}=.3538262 \\
w_{03}^{*}=.1461738, w_{04}^{*}=.1461738\end{array}$ & 36.10842 & 23.21299 & $.1557979 \times 10^{2}$ \\
\hline $55^{\circ}$ & $\begin{array}{l}w_{01}^{*}=.3853710, w_{02}^{*}=.3853710 \\
w_{03}^{*}=.1146290, w_{04}^{*}=.1146290\end{array}$ & 33.06866 & 17.18461 & $.1468697 \times 10^{2}$ \\
\hline $60^{\circ}$ & $\begin{array}{l}w_{01}^{*}=.4000000, w_{02}^{*}=.4000000 \\
w_{03}^{*}=.1000000, w_{04}^{*}=.1000000\end{array}$ & 31.92348 & 15.96174 & $.1566546 \times 10^{2}$ \\
\hline $65^{\circ}$ & $\begin{array}{c}w_{01}^{*}=.4273693, w_{02}^{*}=.4273693 \\
w_{03}^{*}=.0726307, w_{04}^{*}=.0726307\end{array}$ & 32.28743 & 12.99682 & $.1754385 \times 10^{2}$ \\
\hline $70^{\circ}$ & $\begin{array}{l}w_{01}^{*}=.4476615, w_{02}^{*}=.4476615 \\
w_{03}^{*}=.0523384, w_{04}^{*}=.052338\end{array}$ & 33.93088 & 11.61127 & $.2174814 \times 10^{2}$ \\
\hline $75^{\circ}$ & $\begin{array}{l}w_{01}^{*}=.4686287, w_{02}^{*}=.4686587 \\
w_{03}^{*}=.0313712, w_{04}^{*}=.0313712\end{array}$ & 41.88920 & 10.84895 & $.3389167 \times 10^{2}$ \\
\hline
\end{tabular}

We will obtain optimal value of $A_{1}^{*}, A_{2}^{*}, W T^{*}$

Table 2. Output data of two bar truss design problem in using max-additive operator:

The numerical result shows that the optimum weight of the truss is decreased according to increment of angle between two bar and it achieve minimum weight at $55^{\circ}$.After that again its weight become gradually high according to decreasing value of angle between two bar.

DOI: 10.9790/5728-12050197106 $\quad$ www.iosrjournals.org $\quad 105 \mid$ Page




\section{Conclusion}

In this paper a two bar truss design problem has been consideredto minimize its weight in fuzzy environment with fuzzy stress constraint. Optimum weight of the two bar truss result has been obtained by geometric programming followed by Warner's approach .In the test problem the angle between two bar is supplied and examined at which position the structure obtain its optimum weight.

Conflict of interests: The authors declare that there is no conflict of interests.

\section{Acknowledgement}

The research work of MridulaSarkar is financed by Rajiv Gandhi National Fellowship(F1-17.1/201314-SC-wes-42549/(SA-III/Website)), Govt of India.

\section{Reference}

[1]. Zener, C. (1971).Engineering design by geometric programming, Wiley, New York.

[2]. Zimmermann, H.J. (1978).Fuzzy linear programming with several objective function .Fuzzy sets and systems, 1, 45-55.

[3]. Wang, G.Y. \& Wang, W.Q. (1985).Fuzzy optimum design of structure. Engineering Optimization, 8, $291-300$.

[4]. Rao, S. S. (1987). Description and optimum design of fuzzy mechanical systems. Journal of Mechanisms, Transmissions, and Automation in Design, 109(1), 126-132.

[5]. Yeh, Y.C. \& Hsu, D.S. (1990).Structural optimization with fuzzy parameters. Computer and Structure,37(6), 917-924

[6]. Shih, C. J., \& Lee, H. W. (2004). Level-cut approaches of first and second kind for unique solution design in fuzzy engineering optimization problems.Tamkang Journal of Science and Engineering 7(3),189-198.

[7]. Dey, S., \& Roy, T. K. (2014).A Fuzzy programming Technique for Solving Multi-objective Structural Problem. International Journal of Engineering and Manufacturing, 4(5), 24.

[8]. Dey, S. \&Roy, T. K. (2015).Optimum shape design with imprecise coefficients by parametric geometric programming. Decision Science Letter, 4, 407-418.

[9]. Duffin,R.J., Peterson,E.L. \&Zener,C.M. (1967).Geometric programming- theory and applications. Wiley, New York.

[10]. B eightler,C.S.\&Phillips,D.T.(1976).Applied Geometric Programming.Wiley,New York.

[11]. Zadeh, L.A. (1965). Fuzzy set. Information and Control, 8(3), 338-353.

[12]. Cao, B.Y. (1987). Solution and theory of question for a kind of fuzzy positive geometric program.Proceeding of the second IFSA Congress, Tokyo, 1, 205-208.

[13]. Yang, H. \& Cao, B. Y.(2010). Fuzzy geometric programming and its application.Fuzzy information and engineering, 2(1), 101-112.

[14]. Liu, S. T. (2008). Posynomial geometric programming with interval exponents and coefficients. European Journal of Operational Research, 186(1), 17-27.

[15]. Ojha, A. K. \& Das, A.K. (2010).Geometric programming problem with coefficients and exponents associated with binary numbers. International Journal of Computer Science Issues, 7(1), 49-55.

[16]. Dey, S. \&Roy, T. K. (2014).Truss Design Optimization using fuzzy geometric in parametric form. J.Math.Comput.Sci,4(2), 400415.

[17]. Nasseri, S. H., \&Alizadeh, Z. (2014). Optimized solution of a two-bar truss nonlinear problem using fuzzy Geometric programming. Journal of Nonlinear Analysis and Application, 2014. 